\title{
Thermo Portal at the Gotthard Road Tunnel
}

\author{
L.Fischer $^{1}$, C. Durrer ${ }^{1}$, Dr. U. Hoffmann ${ }^{2}$ \\ ${ }^{1}$ ECTN AG, CH-6374 Buochs, Switzerland \\ ${ }^{2}$ DIAS Infrared GmbH, D-01217 Dresden, Germany
}

There have been some devastating tunnel fires in the recent years; one of the most tragic accidents occurred in the Gotthard-Tunnel (2001). The reasons for these incidents vary but vehicle fires caused by technical defects as overheated wheels and engines or the inflammation of payload have triggered tunnel fires in many cases. The observation of cars having symptoms of vehicle fires and preventing them from entering the tunnel could be a measure to avoid many accidents in the future. The aim was to develop an early fire detection system detecting vehicle fires and/or their potential sources outside the tunnel without affecting the running traffic.

A proof of concept realization "Thermo Portal" is done and is running in test mode since Nov. 2010. It is installed at the south portal of Gotthard-Tunnel at an already existing sign gantry (Figure 1). The present posting will give a summary about the concept itself and the first experiences.

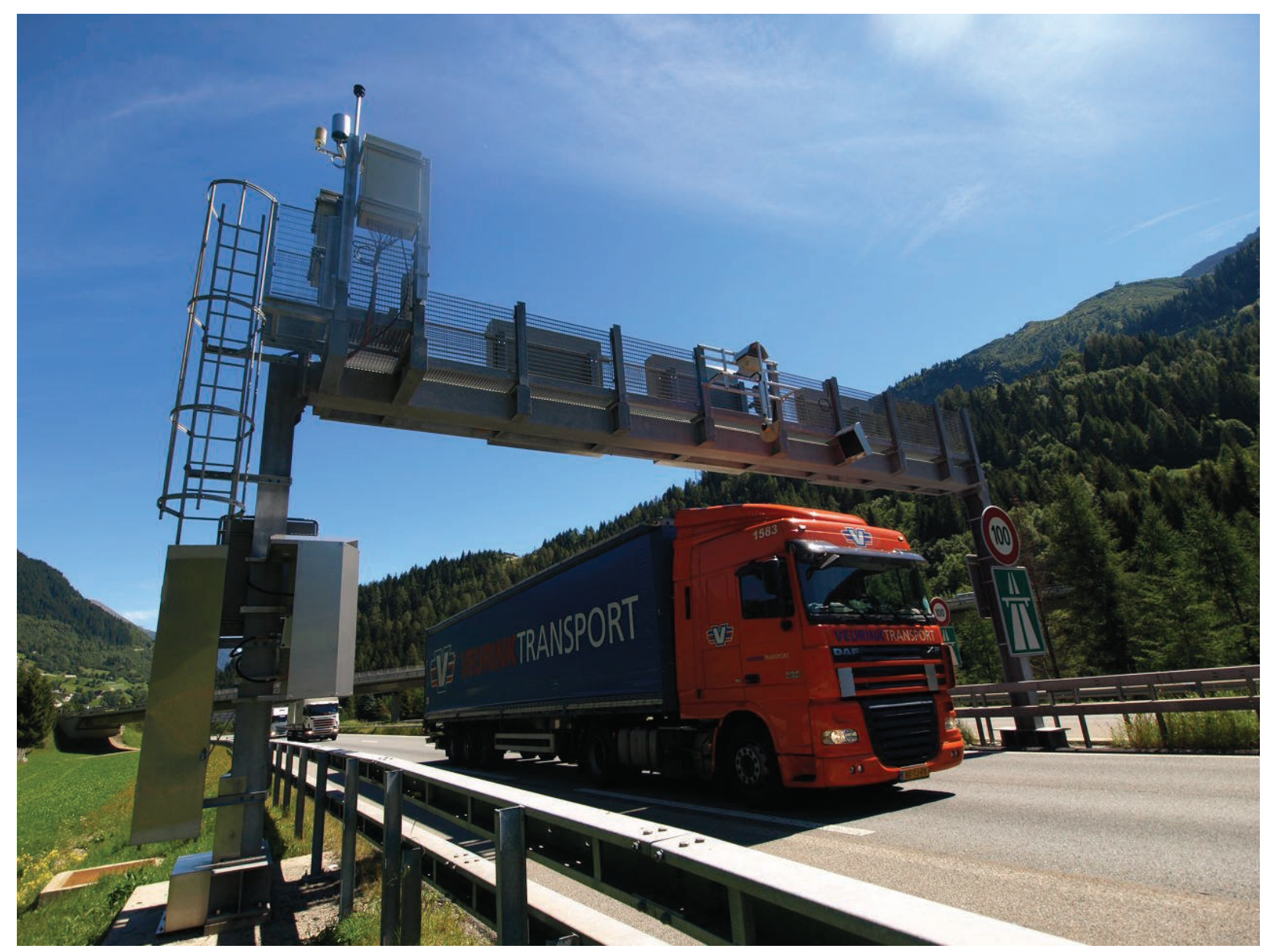

Figure 1: Sign gantry at Gotthard south portal with installed Thermo Portal

The Thermo Portal consists of the combination of 3D laser scanners, video cameras and infrared line cameras mounted on a gantry and the attached PC evaluation software. It is mainly designed to fulfill the following functions: the 3D measurement and the surface temperature measurement of the vehicles passing by as the analysis of the gathered data in many respects:

- vehicle recognition, counting and classification

- registering of vehicle properties

- detection of hot spots 
The laser scanner is used for the creation of a 3D vehicle cubature model as to be seen in Figure 3.

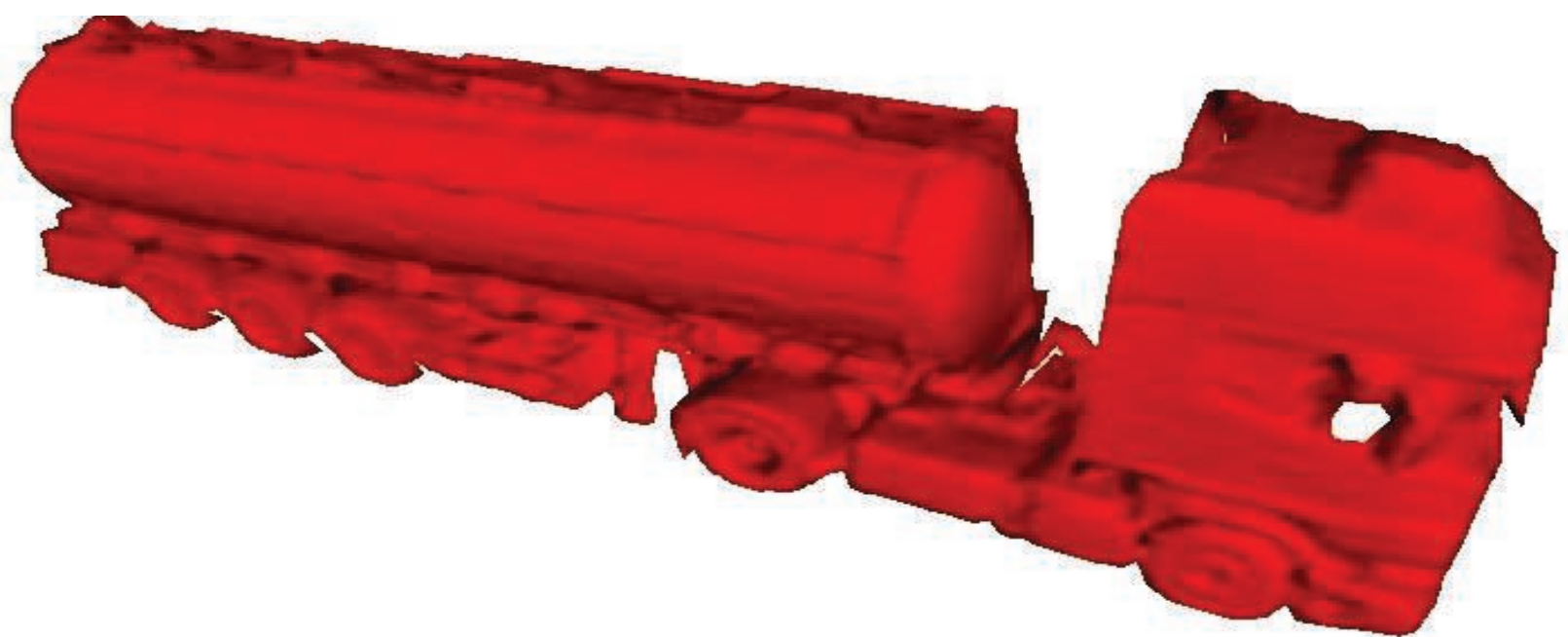

Figure 2: 3D vehicle cubature model

Besides the 3D model any other data as the overall dimensions and the current speed are calculated. An image processing based on all the gained information is designed to recognize the vehicle type (up to 28 different classes). A classification can be done using certain features as:

- vehicle dimensions

- $\quad$ trailer(s)

- number of wheels/axles

- $\quad$ kind of payload (container, vessel etc.)

The complete information is presented on the monitor (Figure 3) and stored in a database system.

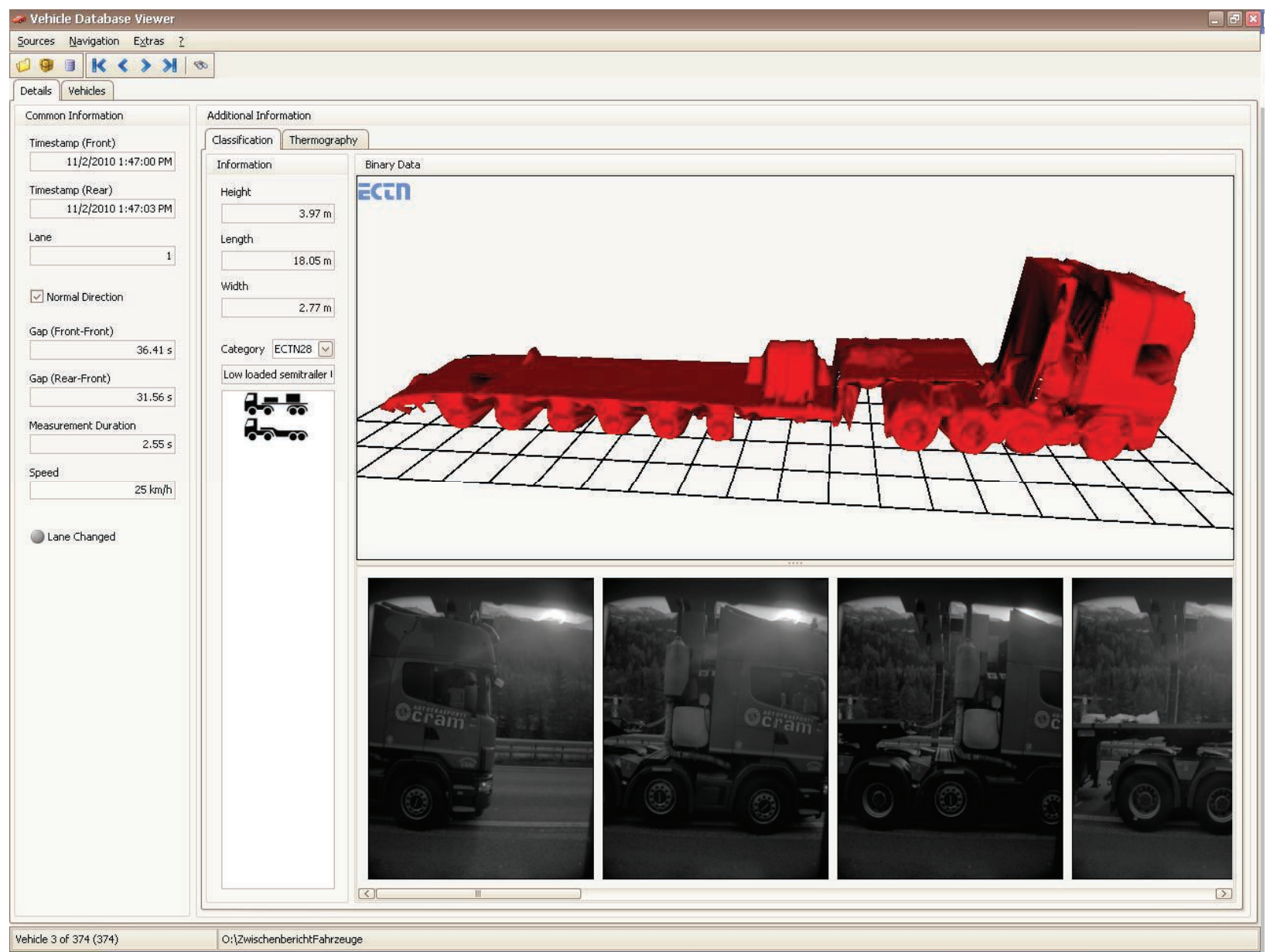

Figure 3: Vehicle information 
The infrared line camera measures a consecutive temperature profile with a constant line frequency. There are two types of cameras introduced:

1. A camera working in the LWIR spectral range $(8 \ldots 14 \mu \mathrm{m})$ and a measurement range from -10 to $400^{\circ} \mathrm{C}$ for monitoring normal surfaces and

2. a camera operating in the MWIR $(3 \ldots 5 \mu \mathrm{m})$ and a resulting measurement range of 350 to $1050^{\circ} \mathrm{C}$ for observing hot parts from the engine.

The sampling frequency vs. the maximum vehicle speed ratio determined the minimal spot size to be resolved. Using line cameras with high line frequencies $(256 \mathrm{~Hz})$ can minimize the risk to miss-out smaller hot spots.

The 3D-model combined with the measured infrared temperature information (Figure 4) delivers finally the data basis for any fire detection analysis.

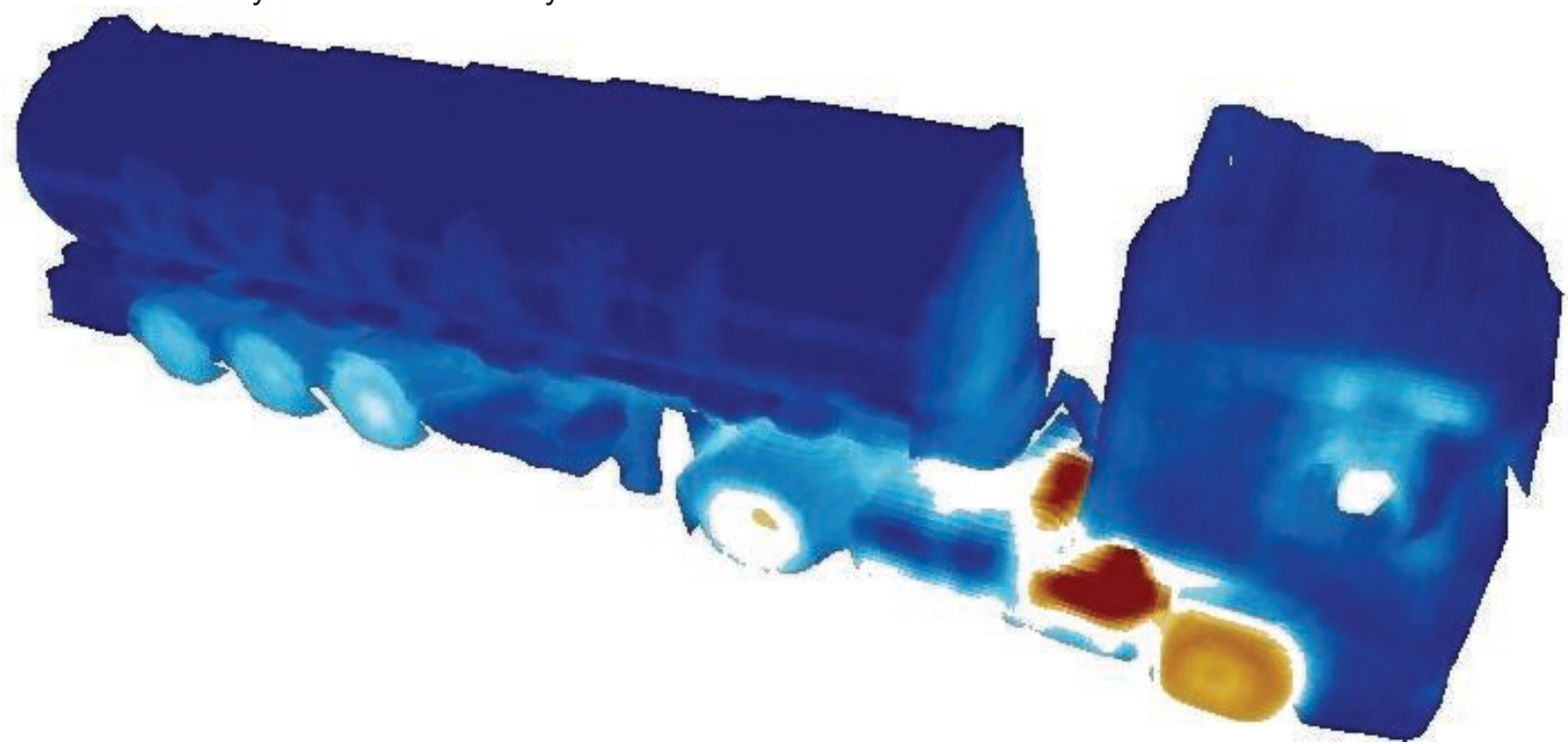

Figure 4: 3D vehicle cubature model with surface temperature overlay texture

The superimposed 3D and infrared data including the local weather information (temperature, humidity, wind and precipitation) provides a fundamental basis to make out the key details to recognize vehicles with a potential risk of car fires. The challenge is to automatically associate the measured temperatures to functional parts of the vehicle, and to apply individual alarm thresholds. The difficulties are as obvious as simple: There are many hot parts at a vehicle without being a potential source of fires such as:

- brake discs

- engine parts incl. turbochargers

- the exhaust system

- reflective parts mirroring the sun

The developed evaluation software must be able to distinguish between different main parts of the vehicle. Critical temperature limits for these parts must be given by the user and/or dynamically calculated dependent on the vehicle classification and weather information. The comparison between acceptable resp. critical and measured temperature values provides additional information presented to the tunnel operator (Figure 5). Currently the data are stored for detailed data evaluation and analysis.

A first, rough evaluation of 570'000 vehicles showed an expected dependency of ambient air temperatures that must be examined further during the warmer months in the future. The automatic segmentation of some vehicle parts works quite well for common parts like cab, wheels or load. Other, more varying hot parts like the exhaust area do require enhancements in the software.

At least one dangerous situation was recorded: One hub on a semitrailer had a temperature of $353^{\circ} \mathrm{C}$.

Otherwise, the infra-red cameras are well protected from contaminations, so no cleaning of the protective glass was necessary during the last three winter months in the Alps.

The final goal is to create an autonomous system able to detect vehicles with (arising) car fires and to inform the traffic control centre for introducing suitable measures such as preventing the vehicle from entering the tunnel or activating an alarm. 


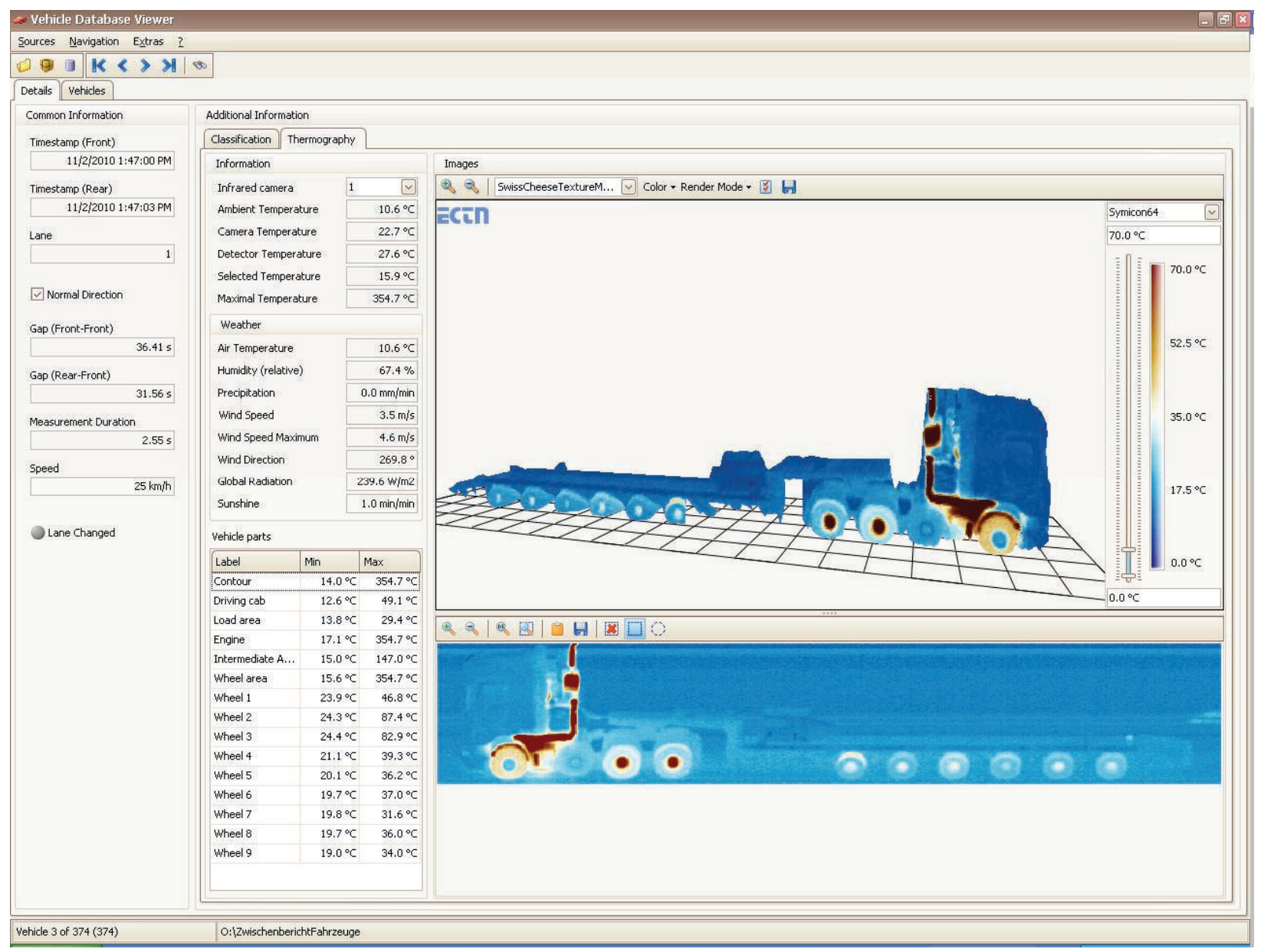

Figure 5: Vehicle temperature data evaluation 